\title{
Antihistamines in Clinical Dentistry offering a Choice for Second Possibility in Reported Cases of Allergy to Local Anesthetics: Report of Two Cases and Literature Review
}

\author{
Shahanavaj Imam Husen Khaji
}

\begin{abstract}
Drug allergy encompasses a spectrum of immunologically mediated hypersensitivity reaction with varying mechanisms and clinical presentation. Type of adverse drug reaction not only affects patient's quality of life, but may also lead to delayed treatment, unnecessary investigations and even mortality.

The most effective strategy for the management of drug allergy is the avoidance or discontinuation of the offending drug. When available, alternative medications with unrelated chemical structures should be substituted. Patients who presents with history of allergy to local anesthetics are common in dental practices. In the present report, retrospective documented history of allergy to local anesthetics (lidocaine) in two patients (50 years/female, 35 years/male) were evaluated critically and needful dental treatment procedures were carried out using antihistamines pheniramine maleate: $22.75 \mathrm{mg} / \mathrm{ml}$; $\mathrm{DPH} \mathrm{HCl}$ : $1 \%)$ as local anesthetic agents. In both the cases, antihistamines proved to be beneficial, effective, devoid of complications.

In conclusion, use of antihistaminic drugs in patients with documented history of allergy could be an alternative drug of choice having local anesthetic properties for minor dental treatment procedures in routine dental practice.
\end{abstract}

Keywords: Local anesthetics, Amide group, Hypersensitivity Antihistamines, Alternative local anesthetic agents.

How to cite this article: Khaji SIH. Antihistamines in Clinical Dentistry offering a Choice for Second Possibility in Reported Cases of Allergy to Local Anesthetics: Report of Two Cases and Literature Review. Int J Head Neck Surg 2014;5(1):35-38.

\section{Source of support $\mathrm{Nil}$}

Conflict of interest None

\section{INTRODUCTION}

$M$ any dental patients claim to be allergic to the commonly used local anesthetic agents. A Iternative methods of treatment, such as general anesthesia, are available but not al ways practical. ${ }^{1}$ The therapeutic use of drugs is common

\footnotetext{
Senior Lecturer

Department of Oral and Maxillofacial Surgery, Tatyasaheb Kore Dental College and Research Centre, Kolhapur Maharashtra, India

Corresponding Author: Shahanavaj Imam Husen Khaji Senior Lecturer, Department of O ral and Maxillofacial Surgery Tatyasaheb Kore Dental College and Research Centre Mahatma Gandhi Hospital Campus, New Pargaon-416137 Kolhapur, Maharashtra, India, Phone: +91-8007055163 e-mail: forevershaan@gmail.com
}

place in dentistry, and the administration of local anesthetics is considered essential whenever potentially painful procedures are contemplated. ${ }^{2}$

Local anesthetics are commonly used drugs. In spite of their widespread use, true hypersensitivity appears to be infrequent. In fact, most of adverse reactions are due to pharmacological, toxic or vasovagal effects of local anesthetics. ${ }^{3}$ Various surveys indicate that the number of deaths attributed to local anesthetic ranges from 1:150,000 to $1: 400,000 .{ }^{4} \mathrm{~W}$ hile adverse reactions to local anesthetics are a reality, a true immunologic reaction to a local anesthetic is rare. ${ }^{5}$

A nesthetic-related allergies, even mild ones, constitute less than $1 \%$ of medical emergencies in the dental office. ${ }^{6}$ M edications are given with the intent of helping a patient, but, unfortunately, nearly all drugs have the potential to cause side effects. Today, there is good evidence in the literature that IgE mediated reactions to pure local anesthetics, particularly to the more commonly used amide group, and are extremely rare. ${ }^{7}$

In this present case report, two patients with retrospective documented history of allergy to lidocaine presented with dental pain and needed minor dental treatment procedures to be done. Hence, authors used antihistamines as alternatives to local anesthetics pheniramine mal eate, diphenhydramine) which proved to be a vital tool for such patients in routine dental practice.

\section{CASE REPORTS}

\section{Case 1}

A 50 years old female patient was referred to the author's hospital for minor oral surgical procedure (extraction of grossly destructed teeth). Past medical and dental history revealed retrospective episode of allergic reaction to local anesthetic agent (lidocaine 2\%; 1:80,000 adrenaline) 20 years earlier. A fter injection of local anesthetic agent, patient experienced episode of itching anand generalized urticaria, pounding in the chest, lightheadedness. No other drugs were administered during the procedure. Patient was hospitalized and kept under ICU setting for 2 to 3 days, where appropriate management (corticosteroids, 
antihistamines) were done accordingly and was labeled allergic to 'CAIN'.

She had insignificant personal anand family history. Her complete blood count, biochemical profiles were normal anand noncontributory. Extraction of 36 was done using 2 cc of antihistaminic drug under local infiltration at the local site. The patient had no pain, no untoward side effects during and after the procedure.

\section{Case 2}

A 35 years old male patient presented for evaluation of a painful teeth in relation to $32,33,34$ region (root stumps) with significant history of drug allergy to lidocaine. U pon detailed elaboration of history retrospective evidence of adverse drug reaction that had occurred 5 years back. Patient was hospitalized and thereafter assiduously avoided local anesthetic injection. Dental extraction was carried out using antihistaminic drug at the regional site. Except for slight pain and tissue irritation during injection patient had a smooth and uneventful recovery.

\section{DISCUSSION}

The relationship between administered drug and adverse reaction, which it may cause, is still the object of extensive research. Currently, adverse drug event according to W HO is defined as 'as any of noxious and unintended effect related with drug administration used in doses recommended, regardless of the route of administration. ${ }^{8}$

Classification of adverse drug reactions into two types are as follows:

- Type A (predictable)-drug overdose, secondary drug effects, side effects, drug interactions.

- Type B (unpredictable)-drug allergy, pseudoallergic/ nonallergic, drug intolerance, drug idiosyncrasy. ${ }^{9}$

A $n$ immunogen (antigen) is a substance capable of eliciting a specific immune response as manifested by specific antibodies or specifically committed lymphocytes by interacting with an antigen-combining site of an antibody. ${ }^{10}$

The major shock organs of drug allergy in humans are as follows:

- The skin (cutaneous)

- Smooth muscle (bronchial, gastrointestinal, vascular)

- Blood cells (hematologic)

- Hepatic, pulmonary, renal and arterial.

The major shock organ in terms of incidence of allergic reactions is the skin, whereas the most life-threatening involves smooth muscle. ${ }^{11}$

Factors altering the immune response to drugs include: age, genetics, frequency and duration of drug administration, route of drug administration, chemical nature of hapten, variable antibody levels. The majority of human allergic reactions are manifested in the skin. Cutaneous allergic drug reactions occur in 2 to $3 \%$ of hospitalized medical service patients.

The complements of immune system are as follows:

- B-lymphocytes

- T-lymphocytes

- Plasma cells (capable of synthesizing five classes of antibodies)

- Macrophages

- Tissue mediators. ${ }^{12}$

The classes of immunoglobulins have specialized functions namely:

- IgG antibodies comprise about $75 \%$ of all antibodies and protect particularly against microorganisms.

- IgA antibodies against foreign substances entering the body.

- IgM antibodies are primarily located intravascularly and are involved in complement reactions.

- IgD antibodies role is unknown. ${ }^{12}$

- IgE antibodies-most significant in drug allergy because of their affinity for mast cells and basophils forms a basis for immediate and anaphylactic allergic reactions. The IgE antibodies comprise only $0.001 \%$ of all antibodies. They are synthesized by plasma cells. ${ }^{13}$

\section{MECHANISMS OF DRUG ALLERGY}

Broadly classified into two headings: immune-mediated reactions and pseudoallergic reactions. Immune-mediated allergic reactions to drugs are classified according to Gell and Coomb's classification system, which describes the predominant immune mechanisms involved in these reactions. $^{11}$

- Typel (IgE mediated): D rug IgE complex binding to mast cells and release of histamine, inflammatory mediators.

- Typell (cytotoxic): Specific IgG/IgM antibodies directed at drug-hapten coated cells.

- Type III (immune complex): Tissue deposition of drug antibody complexes with complement activation and inflammation.

- Type IV (delayed/cell mediated): M HC presentation of drug molecules to $T$ cells with cytokines and inflammatory mediated release may also be associated with activation and recruitment of eosinophils, monocytes and neutrophils.

Unlike immune-mediated drug reaction, pseudoallergic reactions are not associated with the production of antibodies or sensitized T cells. ${ }^{14}$

A Iternatives for ' $C A$ IN' sensitive patients are as follows:

- Benzyl alcohol

- Normal saline solution 


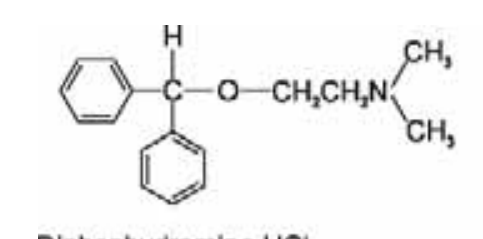

Diphenhydramine $\mathrm{HCl}$ Pheniramine maleate

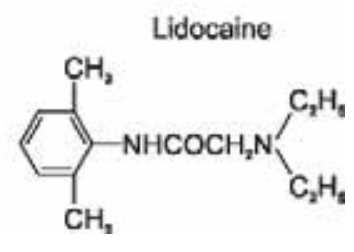

Fig. 1: Chemical configurations of commonly used antihistamines and LAs

- Antihistamines as local anesthetics (LAs) (Fig. 1)

- General anesthesia

- IV sedation (diazepam, pentobarbital, meperidine, droperidol, fentanyl)

- Nitrous oxide-oxygen therapy. ${ }^{15-17}$

The entire group of drugs classified as antihistamines possess local anesthetic properties has been known since 1942 when first reported by Halpern. ${ }^{18}$ One of the earliest reports of the use of diphenhydramine, pheniramine mal eate as local anesthetics since 1956. Rosanov reported a series of 200 cases of minor skin surgery using antihistamines and stated that the advantages as a local anesthetic agentnegligible toxicity, allergic reactions to their use are extremely rare or absent, rapid onset of action with a potent local anesthetic property. ${ }^{19}$

In 1964, successful extraction of maxillary teeth was carried out using antihistamines as local anesthetics by Smith and Campolattaro.

Welborn and $K$ ane reported first series of mandibular blocks using $1 \%$ diphenhydramine in combination with 1:100,000 epinephrine for various dental treatment procedures. A ntihistamines are described as agents that possess anticholinergic (antispasmodic), antiemetic and sedative effects. ${ }^{20}$ D rawbacks of antihistamines used as local anesthetics are as follows:

- Confusion

- Nausea

- Diplopia

- Dryness of the mouth, throat, nose

- Headache

- Urticaria

- Burning sensation upon injection

- A naphylactic shock (rare). ${ }^{1}$

Relative contraindications of antihistamines include: pregnancy, nursing mothers, asthma, narrow angle glaucoma, peptic ulcer, benign prostatic hypertrophy, obstructive bowel disease. ${ }^{21}$

In our experience of two reported cases, with the use of antihistaminic drugs, has revealed to be a valuable and highly effective agent in cases of hypersensitivity to local anesthetics. However, more and more frequently members of dental profession are being called upon to treat patients who claim to be allergic. A lthough general anesthesia is one of the al ternatives to local anesthetics, a suitable replacement for the common local anesthetic exists in the form of certain antihistamines which hold true with the presented cases.

\section{CONCLUSION}

It is important that clinicians must be able to evaluate a suspected allergic patient so that appropriate management can be made.

$M$ any dental patients claim to be allergic to local anesthetics used in day-to-day routine dental practice. Although, alternativs modalities of treatment are available, such as GA, but it is not al ways practical. Injectable antihistamines (local anesthetic properties of the antihistamines) used in the present paper havebeen proved beneficial, a reasonablealternative, safe, inexpensiveand effectivelocal anesthetic agentfor minor dental treatment procedures in documented cases of hypersensitivity to local anesthetics.

\section{REFERENCES}

1. M alamed SF. Diphenhydramine hydrochloride its use as a local anesthetic in dentistry. A nesth Prog 1973; M ay-J un;20(3)e: 76-82.

2. Malamed SF. Handbook of local anesthesia. 4th ed. Saunders 2002;303-304.

3. LukawskaJ, Carbellero M R, Tsabouri S, DugueE. Hypersensitivity to local anesthetics- 6 facts and 7 myths. Curt Allergy Clin Immunol 2009 Aug;22(3):117-120.

4. Lu DP. M anaging patients with LA complications using alternative methods. Pa Dent J $2002 \mathrm{M}$ ay-J un;69(3):22-29.

5. Eggleston $S T$, L ush LW. U nderstanding allergic reactions to local anesthetics. A nn Pharmacother 1996 J ul-A ug;30(7-8):851-857.

6. Isen D. Point of care. J Canadian D ent A ssoc $2005 \mathrm{M}$ ar; 71(3): 193-194.

7. M acy E. L ocal anesthetic adverse reaction evaluation: the role of the allergist. A n A llergy Clin Immunol 20030 ct;91(4):314-315.

8. Gomulka K, Paruzynska A, Wolanczyk MA, Medrala W. Hypersensitivity to lidocaine-retrospective case series. Int Rev Allergol Clin Immunol 2010;16(3-4):90-93.

9. K han DA, Solensky R. Drug allergy. J Allergy Clin Immunol 2010 Feb;125(2 Suppl 2):S126-137.

10. Park BK, Coleman JW, Kitteringham NR. Drug disposition anand drug hypersensitivity. Biochem Pharmacol $1987 \mathrm{M}$ ar; 36(5):581-590.

11. Gell PGH, Coombs RRA. Clinical aspects of immunology. 3rd ed. Oxford; B lackwell Scientific Publication; 1975.

12. Witte KW, West DP. Immunology of adverse drug reactions. Pharmacotherapy 1982 J an-F eb;2(1):54-65.

13. Buisseret PD. Allergy. Sci A m 1982 A ug;247(2):86-95.

14. Sylvia LM. Drug allergy and pseudoallergy. In: Tisdale JE, M iller DA, editors. D rug-induced diseases: prevention, detection and management. B ethesda, M D: A merican Society of HealthSystem Pharmacists 2005;27-55. 
15. B artfeild JM, Jandreau SW, Raccio RN. Randomised trial of DPH versus benzyl alcohol with epinephrine as an alternative to lidocaine. Ann Emerg M ed $1998 \mathrm{Dec} ; 32(6)$ : 650-654.

16. Wightman MA, Vaughan RW. Comparison of compounds used for intradermal anesthesia. A nesthesiology 1976 Dec;45(6): 687-689.

17. Peter G, Pavlidakey M D, E rin EB , Stephen EH. Diphenhydramine as an alternative local anesthetic agent- a case report. J Clin A esthetic Dermatol 2009 0ct;2(10)37-40.
18. Halpern BN. Les antihistaminiques de synthese. A rch Inter Pharmacodyn et de Therap 1942;68:339-408.

19. Rosanove R. Local anesthesia for allergic patients: the use of diphenhydramine hydrochlorid as a local anesthetic agent. M ed J A ust 1963 A pr;1:613-614.

20. Welborn JF, Kane JP. Conduction anesthesia using diphenhydramine hydrochloride. J A m D entA ss 1964 D ec;69:706-709.

21. U ckan S, Guler N, Surner M , U ngor M . L ocal anesthetic efficacy for oral surgery: comparison of DPH and prilocaine. Oral Surg Oral M ed Oral Pathol Oral Radiol Endo 1998 J ul;86(1):26-30. 